EUROPEAN ORGANIZATION FOR NUCLEAR RESEARCH

CERN-PPE/96-168

22nd November 1996

\title{
Inclusive Production of Neutral Pions
}

\section{in Hadronic Z Decays}

The ALEPH Collaboration

\begin{abstract}
A measurement of the inclusive production of $\pi^{0}$ mesons in hadronic $\mathrm{Z}$ decays is presented and compared to Monte Carlo model predictions. The analysis is based on approximately 2 million hadronic events recorded with the ALEPH detector at LEP at a centre-of-mass energy of $\sqrt{s}=91.2 \mathrm{GeV}$. Neutral pions are reconstructed using photons measured in the electromagnetic calorimeter and photons from conversion pairs. The inclusive $\pi^{0}$ momentum spectrum is measured in the range $0.025<x_{p}=p / p_{\text {beam }}<1$ to be $4.80 \pm 0.07$ (stat) \pm 0.31 (sys). The differential inclusive $\pi^{0}$ cross section is also measured as a function of transverse momentum with respect to the event plane $\left(p_{\mathrm{T}}^{\text {in }}\right.$ and $\left.p_{\mathrm{T}}^{\text {out }}\right)$.
\end{abstract}

(To be submitted to Z. Phys. C.) 


\section{The ALEPH Collaboration}

R. Barate, D. Buskulic, D. Decamp, P. Ghez, C. Goy, J.-P. Lees, A. Lucotte, M.-N. Minard, J.-Y. Nief, P. Odier,

B. Pietrzyk

Laboratoire de Physique des Particules (LAPP), IN $P^{3}$-CNRS, 74019 Annecy-le-Vieux Cedex, France

M.P. Casado, M. Chmeissani, P. Comas, J.M. Crespo, M. Delfino, E. Fernandez, M. Fernandez-Bosman, Ll. Garrido, ${ }^{15}$ A. Juste, M. Martinez, S. Orteu, C. Padilla, I.C. Park, A. Pascual, J.A. Perlas, I. Riu, F. Sanchez, F. Teubert

Institut de Fisica d'Altes Energies, Universitat Autonoma de Barcelona, 08193 Bellaterra (Barcelona), Spain ${ }^{7}$

A. Colaleo, D. Creanza, M. de Palma, G. Gelao, M. Girone, G. Iaselli, G. Maggi, M. Maggi, N. Marinelli, S. Nuzzo, A. Ranieri, G. Raso, F. Ruggieri, G. Selvaggi, L. Silvestris, P. Tempesta, A. Tricomi, ${ }^{3}$ G. Zito

Dipartimento di Fisica, INFN Sezione di Bari, 70126 Bari, Italy

X. Huang, J. Lin, Q. Ouyang, T. Wang, Y. Xie, R. Xu, S. Xue, J. Zhang, L. Zhang, W. Zhao

Institute of High-Energy Physics, Academia Sinica, Beijing, The People's Republic of China ${ }^{8}$

D. Abbaneo, R. Alemany, A.O. Bazarko, P. Bright-Thomas, M. Cattaneo, F. Cerutti, P. Coyle, H. Drevermann, R.W. Forty, M. Frank, R. Hagelberg, J. Harvey, P. Janot, B. Jost, E. Kneringer, J. Knobloch, I. Lehraus, G. Lutters, P. Mato, A. Minten, R. Miquel, Ll.M. Mir, ${ }^{2}$ L. Moneta, T. Oest, ${ }^{20}$ A. Pacheco, J.-F. Pusztaszeri, F. Ranjard, P. Rensing, ${ }^{12}$ G. Rizzo, L. Rolandi, D. Schlatter, M. Schmelling, ${ }^{24}$ M. Schmitt, O. Schneider, W. Tejessy, I.R. Tomalin, A. Venturi, H. Wachsmuth, A. Wagner

European Laboratory for Particle Physics (CERN), 1211 Geneva 23, Switzerland

Z. Ajaltouni, A. Barrès, C. Boyer, A. Falvard, P. Gay, C . Guicheney, P. Henrard, J. Jousset, B. Michel, S. Monteil, J-C. Montret, D. Pallin, P. Perret, F. Podlyski, J. Proriol, P. Rosnet, J.-M. Rossignol

Laboratoire de Physique Corpusculaire, Université Blaise Pascal, IN ${ }^{2} P^{3}$-CNRS, Clermont-Ferrand, 63177 Aubière, France

T. Fearnley, J.B. Hansen, J.D. Hansen, J.R. Hansen, P.H. Hansen, B.S. Nilsson, B. Rensch, A. Wäänänen

Niels Bohr Institute, 2100 Copenhagen, Denmark ${ }^{9}$

G. Daskalakis, A. Kyriakis, C. Markou, E. Simopoulou, I. Siotis, A. Vayaki, K. Zachariadou

Nuclear Research Center Demokritos (NRCD), Athens, Greece

A. Blondel, G. Bonneaud, J.C. Brient, P. Bourdon, A. Rougé, M. Rumpf, A. Valassi, ${ }^{6}$ M. Verderi, H. Videau Laboratoire de Physique Nucléaire et des Hautes Energies, Ecole Polytechnique, IN ${ }^{2} P^{3}$-CNRS, 91128 Palaiseau Cedex, France

D.J. Candlin, M.I. Parsons

Department of Physics, University of Edinburgh, Edinburgh EH9 3JZ, United Kingdom ${ }^{10}$

E. Focardi, ${ }^{21}$ G. Parrini

Dipartimento di Fisica, Università di Firenze, INFN Sezione di Firenze, 50125 Firenze, Italy

M. Corden, C. Georgiopoulos, D.E. Jaffe

Supercomputer Computations Research Institute, Florida State University, Tallahassee, FL 32306-4052, USA

A. Antonelli, G. Bencivenni, G. Bologna, ${ }^{4}$ F. Bossi, P. Campana, G. Capon, D. Casper, V. Chiarella, G. Felici, P. Laurelli, G. Mannocchi, ${ }^{5}$ F. Murtas, G.P. Murtas, L. Passalacqua, M. Pepe-Altarelli

Laboratori Nazionali dell'INFN (LNF-INFN), 00044 Frascati, Italy

L. Curtis, S.J. Dorris, A.W. Halley, I.G. Knowles, J.G. Lynch, V. O'Shea, C. Raine, J.M. Scarr, K. Smith, P. Teixeira-Dias, A.S. Thompson, E. Thomson, F. Thomson, R.M. Turnbull

Department of Physics and Astronomy, University of Glasgow, Glasgow G12 8QQ,United Kingdom ${ }^{10}$ 
U. Becker, C. Geweniger, G. Graefe, P. Hanke, G. Hansper, V. Hepp, E.E. Kluge, A. Putzer, M. Schmidt, J. Sommer, H. Stenzel, K. Tittel, S. Werner, M. Wunsch

Institut für Hochenergiephysik, Universität Heidelberg, 69120 Heidelberg, Fed. Rep. of Germany ${ }^{16}$

R. Beuselinck, D.M. Binnie, W. Cameron, P.J. Dornan, E.B. Martin, A. Moutoussi, J. Nash, J.K. Sedgbeer, A.M. Stacey, M.D. Williams

Department of Physics, Imperial College, London SW7 2BZ, United Kingdom ${ }^{10}$

G. Dissertori, P. Girtler, D. Kuhn, G. Rudolph

Institut für Experimentalphysik, Universität Innsbruck, 6020 Innsbruck, Austria ${ }^{18}$

A.P. Betteridge, C.K. Bowdery, P. Colrain, G. Crawford, A.J. Finch, F. Foster, G. Hughes, T. Sloan, M.I. Williams

Department of Physics, University of Lancaster, Lancaster LA1 4YB, United Kingdom ${ }^{10}$

A. Galla, I. Giehl, A.M. Greene, C. Hoffmann, K. Jakobs, K. Kleinknecht, G. Quast, B. Renk, E. Rohne, H.G. Sander, P. van Gemmeren, C. Zeitnitz

Institut für Physik, Universität Mainz, 55099 Mainz, Fed. Rep. of Germany ${ }^{16}$

J.J. Aubert, C. Benchouk, A. Bonissent, G. Bujosa, D. Calvet, J. Carr, C. Diaconu, F. Etienne, N. Konstantinidis, O. Leroy, P. Payre, D. Rousseau, M. Talby, A. Sadouki, M. Thulasidas, K. Trabelsi

Centre de Physique des Particules, Faculté des Sciences de Luminy, IN ${ }^{2} P^{3}$-CNRS, 13288 Marseille, France

M. Aleppo, F. Ragusa ${ }^{21}$

Dipartimento di Fisica, Università di Milano e INFN Sezione di Milano, 20133 Milano, Italy

R. Berlich, W. Blum, V. Büscher, H. Dietl, F. Dydak, ${ }^{21}$ G. Ganis, C. Gotzhein, H. Kroha, G. Lütjens, G. Lutz, W. Männer, H.-G. Moser, R. Richter, A. Rosado-Schlosser, S. Schael, R. Settles, H. Seywerd, R. St. Denis, H. Stenzel, W. Wiedenmann, G. Wolf

Max-Planck-Institut für Physik, Werner-Heisenberg-Institut, 80805 München, Fed. Rep. of Germany ${ }^{16}$

J. Boucrot, O. Callot, ${ }^{21}$ S. Chen, Y. Choi, ${ }^{26}$ A. Cordier, M. Davier, L. Duflot, J.-F. Grivaz, Ph. Heusse, A. Höcker, A. Jacholkowska, M. Jacquet, D.W. Kim ${ }^{19}$ F. Le Diberder, J. Lefrançois, A.-M. Lutz, I. Nikolic, H.J. Park, ${ }^{19}$ M.-H. Schune, S. Simion, J.-J. Veillet, I. Videau, D. Zerwas

Laboratoire de l'Accélérateur Linéaire, Université de Paris-Sud, IN ${ }^{2} P^{3}-C N R S, 91405$ Orsay Cedex, France

P. Azzurri, G. Bagliesi, G. Batignani, S. Bettarini, C. Bozzi, G. Calderini, M. Carpinelli, M.A. Ciocci, V. Ciulli, R. Dell'Orso, R. Fantechi, I. Ferrante, L. Foà, ${ }^{1}$ F. Forti, A. Giassi, M.A. Giorgi, A. Gregorio, F. Ligabue, A. Lusiani, P.S. Marrocchesi, A. Messineo, F. Palla, G. Sanguinetti, A. Sciabà, P. Spagnolo, J. Steinberger, R. Tenchini, G. Tonelli, ${ }^{25}$ C. Vannini, P.G. Verdini

Dipartimento di Fisica dell'Università, INFN Sezione di Pisa, e Scuola Normale Superiore, 56010 Pisa, Italy

G.A. Blair, L.M. Bryant, J.T. Chambers, Y. Gao, M.G. Green, T. Medcalf, P. Perrodo, J.A. Strong, J.H. von Wimmersperg-Toeller

Department of Physics, Royal Holloway \& Bedford New College, University of London, Surrey TW20 OEX, United Kingdom ${ }^{10}$

D.R. Botterill, R.W. Clifft, T.R. Edgecock, S. Haywood, P. Maley, P.R. Norton, J.C. Thompson, A.E. Wright Particle Physics Dept., Rutherford Appleton Laboratory, Chilton, Didcot, Oxon OX11 OQX, United Kingdom ${ }^{10}$

B. Bloch-Devaux, P. Colas, S. Emery, W. Kozanecki, E. Lançon, M.C. Lemaire, E. Locci, P. Perez, J. Rander, J.-F. Renardy, A. Roussarie, J.-P. Schuller, J. Schwindling, A. Trabelsi, B. Vallage

CEA, DAPNIA/Service de Physique des Particules, CE-Saclay, 91191 Gif-sur-Yvette Cedex, France ${ }^{17}$

S.N. Black, J.H. Dann, R.P. Johnson, H.Y. Kim, A.M. Litke, M.A. McNeil, G. Taylor

Institute for Particle Physics, University of California at Santa Cruz, Santa Cruz, CA 95064, USA ${ }^{22}$

C.N. Booth, R. Boswell, C.A.J. Brew, S. Cartwright, F. Combley, M.S. Kelly, M. Letho, W.M. Newton, J. Reeve, L.F. Thompson

Department of Physics, University of Sheffield, Sheffield S3 7RH, United Kingdom ${ }^{10}$

A. Böhrer, S. Brandt, G. Cowan, C. Grupen, J. Minguet-Rodriguez, F. Rivera, P. Saraiva, L. Smolik, F. Stephan, 
Fachbereich Physik, Universität Siegen, 57068 Siegen, Fed. Rep. of Germany ${ }^{16}$

M. Apollonio, L. Bosisio, R. Della Marina, G. Giannini, B. Gobbo, G. Musolino

Dipartimento di Fisica, Università di Trieste e INFN Sezione di Trieste, 34127 Trieste, Italy

J. Rothberg, S. Wasserbaech

Experimental Elementary Particle Physics, University of Washington, WA 98195 Seattle, U.S.A.

S.R. Armstrong, P. Elmer, Z. Feng, ${ }^{27}$ D.P.S. Ferguson, Y.S. Gao, ${ }^{23}$ S. González, J. Grahl, T.C. Greening, O.J. Hayes, H. Hu, P.A. McNamara III, J.M. Nachtman, W. Orejudos, Y.B. Pan, Y. Saadi, I.J. Scott, J. Walsh, Sau Lan Wu, X. Wu, J.M. Yamartino, M. Zheng, G. Zobernig

Department of Physics, University of Wisconsin, Madison, WI 53706, USA ${ }^{11}$

\footnotetext{
${ }^{1}$ Now at CERN, 1211 Geneva 23, Switzerland.

${ }^{2}$ Supported by Dirección General de Investigación Científica y Técnica, Spain.

${ }^{3}$ Also at Dipartimento di Fisica, INFN, Sezione di Catania, Catania, Italy.

${ }^{4}$ Also Istituto di Fisica Generale, Università di Torino, Torino, Italy.

${ }^{5}$ Also Istituto di Cosmo-Geofisica del C.N.R., Torino, Italy.

${ }^{6}$ Supported by the Commission of the European Communities, contract ERBCHBICT941234.

${ }^{7}$ Supported by CICYT, Spain.

${ }^{8}$ Supported by the National Science Foundation of China.

${ }^{9}$ Supported by the Danish Natural Science Research Council.

${ }^{10}$ Supported by the UK Particle Physics and Astronomy Research Council.

${ }^{11}$ Supported by the US Department of Energy, grant DE-FG0295-ER40896.

${ }^{12}$ Now at Dragon Systems, Newton, MA 02160, U.S.A.

${ }^{13}$ Supported by the US Department of Energy, contract DE-FG05-92ER40742.

${ }^{14}$ Supported by the US Department of Energy, contract DE-FC05-85ER250000.

${ }^{15}$ Permanent address: Universitat de Barcelona, 08208 Barcelona, Spain.

${ }^{16}$ Supported by the Bundesministerium für Bildung, Wissenschaft, Forschung und Technologie, Fed. Rep. of Germany.

${ }^{17}$ Supported by the Direction des Sciences de la Matière, C.E.A.

${ }^{18}$ Supported by Fonds zur Förderung der wissenschaftlichen Forschung, Austria.

${ }^{19}$ Permanent address: Kangnung National University, Kangnung, Korea.

${ }^{20}$ Now at DESY, Hamburg, Germany.

${ }^{21}$ Also at CERN, 1211 Geneva 23, Switzerland.

${ }^{22}$ Supported by the US Department of Energy, grant DE-FG03-92ER40689.

${ }^{23}$ Now at Harvard University, Cambridge, MA 02138, U.S.A.

${ }^{24}$ Now at Max-Plank-Institüt für Kernphysik, Heidelberg, Germany.

${ }^{25}$ Also at Istituto di Matematica e Fisica, Università di Sassari, Sassari, Italy.

${ }^{26}$ Permanent address: Sung Kyun Kwan University, Suwon, Korea.

${ }^{27}$ Now at The Johns Hopkins University, Baltimore, MD 21218, U.S.A.
} 


\section{Introduction}

The particle composition of hadronic final states in $\mathrm{e}^{+} \mathrm{e}^{-}$annihilation has been an important subject of study at the LEP collider as well as at other $\mathrm{e}^{+} \mathrm{e}^{-}$storage rings. The measurement of inclusive particle production rates provides information about hadronization, where quarks and gluons are transformed into colour-neutral hadrons. This takes place at low momentum transfers and is thus not calculable by perturbative QCD. Instead, it must be described by QCD-motivated hadron production models implemented as Monte Carlo event generators.

Previous studies of particle composition of hadronic $\mathrm{Z}$ decays include measurements of charged particles (mainly $\pi^{ \pm}, \mathrm{K}^{ \pm}$, and $(\mathrm{p}, \overline{\mathrm{p}})$ ) $[1,2,3,4]$ the $\eta$ and $\eta^{\prime}$ mesons [5, 6] and vector mesons $[7,8,9]$. The major part of neutral energy is carried by neutral pions, which are the subject of this analysis.

With the large number of hadronic events recorded by the ALEPH detector at a centreof-mass energy of $\sqrt{s}=91.2 \mathrm{GeV}$ during the 1992-1994 running period of LEP, an accurate measurement of the inclusive $\pi^{0}$ production rate in the $x_{p}$ range from 0.025 to 1 can be performed. Aspects of the ALEPH detector relevant to this analysis are given in Section 2, and the event selection is described in Section 3.

In Sections 4 and 5, the measurement technique is described. Neutral pions are reconstructed from their decays into two photons. These photons can be measured either as neutral clusters in the electromagnetic calorimeter ("calorimetric photons") or by their conversion into an electron-positron pair ("converted photons") detected in the tracking chambers. At low energy $\left(E_{\gamma}<5 \mathrm{GeV}\right)$, where most of the photons are produced, the determination of the photon energy for converted photons is more accurate than for calorimetric photons because of the very good resolution for track momenta.

Given enough data, the most accurate measurement would be based on $\pi^{0}$ mesons in which both photons produce conversion pairs. The probability for a photon to convert within the ALEPH tracking system is, however, only about $7 \%$, so that only a very small fraction of the $\pi^{0}$ mesons fall into this category. With the available data sample, the measurement with two conversions can only be carried out using four very broad momentum bins. This measurement is presented in Section 5.1.

In order to obtain more detail information on the shape of the momentum spectrum, the analysis has also been carried out using one conversion photon combined with one calorimetric photon. Since the fraction of $\pi^{0}$ mesons in this category is significantly larger, a finer binning in momentum can be used. As will be discussed in Section 5.2, this technique requires a knowledge of the photon detection efficiency in the calorimeter. This is difficult to determine with high precision in a hadronic environment. A calibration procedure based on the comparison of the $\pi^{0}$ rate using two conversions with that using one conversion and one calorimetric photon is applied. The final result for the momentum spectrum thus combines these two methods. The $\pi^{0}$ production rate is measured as a function of the momentum and of the transverse momentum in and out of the event plane, $p_{\mathrm{T}}^{\text {in }}$ and $p_{\mathrm{T}}^{\text {out }}$.

As a cross check, an analysis was also performed for $\pi^{0}$ mesons which are reconstructed via two calorimetric photons. This is presented in Section 5.3.

In Section 6 the $\pi^{0}$ spectra are compared to Monte Carlo predictions from JETSET 7.4 [10], which is based on the Lund string model, and HERWIG 5.8 [11], based on a cluster model. The momentum spectrum is compared to corresponding measurements of charged pions [1]. From isospin conservation one would expect half as many neutral pions as charged pions. Isospin violating effects are not negligible, however, and these are found to be well described by Monte Carlo models. The $p_{\mathrm{T}}$ spectra of neutral pions are compared to the $p_{\mathrm{T}}$ spectra of charged particles [2], where some deviations from model predictions are found. 


\section{The ALEPH detector}

The ALEPH detector and its performance are described in detail elsewhere [12]. The present analysis mainly uses the outer tracking components and the electromagnetic calorimeter.

Charged particles are measured over the polar angle range $|\cos \theta|<0.966$ by the cylindrical inner drift chamber (ITC), with eight drift chamber layers, and the large cylindrical time projection chamber (TPC), which provides up to 21 space points and up to 338 measurements of the ionization energy loss $(\mathrm{d} E / \mathrm{d} x)$. These chambers are located in a magnetic field of $1.5 \mathrm{~T}$ and together measure the momentum of charged particles with a resolution of $\delta p_{\mathrm{T}} / p_{\mathrm{T}}=$ $0.0008 p_{\mathrm{T}}(\mathrm{GeV} / c)^{-1} \oplus 0.005$.

The TPC is surrounded by the electromagnetic calorimeter (ECAL), which covers the angular range $|\cos \theta|<0.98$ and has a thickness of 22 radiation lengths $\left(X_{0}\right)$. It is finely segmented in projective towers of approximately $0.9^{\circ}$ by $0.9^{\circ}$; this results in an angular resolution of $\sigma_{\theta, \phi}=$ $2.5 \mathrm{mrad} / \sqrt{E / \mathrm{GeV}} \oplus 0.25 \mathrm{mrad}$. Each tower is read out in three segments of depth (storeys) with four, nine and nine radiation lengths. The energy resolution is $\sigma_{E} / E=0.18 / \sqrt{E / \mathrm{GeV}}+0.009$ for electron induced showers and $\sigma_{E} / E \approx 0.25 / \sqrt{E / \mathrm{GeV}}$ for photons in a hadronic environment (cf. Section 4.2).

For the reconstruction of photons via conversion, it is important to know accurately the amount of material in the detector. The central region of the beam pipe is made of beryllium and has $0.003 X_{0}$. Surrounding the beam pipe, the silicon vertex detector (VDET) consists of two concentric layers, which together have a thickness of $0.041 X_{0}$. The amount of material from the inner and outer ITC wall is $0.003 X_{0}$ and $0.01 X_{0}$, respectively, and the inner TPC wall has $0.023 X_{0}$.

Thus the total amount of material between the beam line and the TPC corresponds to approximately $8 \%$ of a radiation length for tracks at normal incidence. Based only on a knowledge of the materials used, which are in some components complicated and inhomogeneous, the total number of radiation lengths would be difficult to estimate accurately. The amount of material must be known as precisely as possible, however, since it directly influences the probability for a photon to convert. A more accurate determination is obtained using a calibration procedure based on photon conversions in the TPC gas; this is described in Section 4.1.

\section{Event selection and simulation}

The selection of hadronic events is based on charged particle tracks. Good tracks are required to originate from the interaction region (with transverse impact parameter $\left|d_{0}\right|<2 \mathrm{~cm}$ and longitudinal impact parameter $\left|z_{0}\right|<5 \mathrm{~cm}$ ), to have at least four TPC hits, to be within a polar angle range of $20^{\circ}<\theta<160^{\circ}$, and to have a transverse momentum with respect to the beam line of $p_{\mathrm{T}}>200 \mathrm{MeV} / c$. Events are selected which contain at least five good tracks and for which the total energy carried by these tracks exceeds $15 \mathrm{GeV}$. In order to select fully contained events, the sphericity axis must be in the range $35^{\circ}<\theta_{\mathrm{sph}}<145^{\circ}$.

With these cuts, 2171204 events are selected from the 1992-1994 running period. The largest background in this event sample arises from $\tau^{+} \tau^{-}$events and is estimated to be $0.3 \%$.

For determining the detector acceptance and the efficiencies, samples of events were generated with the Jetset Monte Carlo. The program was modified to include improved charm and bottom branching ratios and a generation of prompt photons using DYMU3 [14]. These events were passed through the full detector simulation and reconstruction program and were analysed in the same way as the data. The important parameters of the generator were tuned to describe the ALEPH data using inclusive charged particle distributions and distributions of event-shape variables [2]. 


\section{Photon reconstruction}

Two methods of photon identification are used in this analysis: photon reconstruction from $\gamma$ conversion into an $\mathrm{e}^{+} \mathrm{e}^{-}$pair in the material of the ALEPH detector, and from neutral clusters in the electromagnetic calorimeter.

\subsection{Photon reconstruction from pair conversions}

In the search for $\mathrm{e}^{+} \mathrm{e}^{-}$pairs, tracks of electron candidates are required to have at least four TPC hits, a polar angle in the range $20^{\circ}<\theta<160^{\circ}$, and a transverse momentum $p_{\mathrm{T}}>200 \mathrm{MeV} / c$. In order to discriminate between electrons and other charged particles, the $\mathrm{d} E / \mathrm{d} x$ and the shape of the electromagnetic shower in the calorimeter are used. The measured values of these quantities minus the expected value for an electron, divided by the corresponding resolution, yields the quantities $R_{\mathrm{I}}, R_{\mathrm{T}}$ and $R_{\mathrm{L}}$ for ionization, transverse shape and longitudinal shape, respectively. Tracks are classified as electron tracks if they fulfill the following requirements:

- $\left|R_{\mathrm{I}}\right| \leq 3$, for tracks with $\mathrm{d} E / \mathrm{d} x$ measurement on at least 35 wires;

- $R_{\mathrm{T}} \geq-3$, for $p>1 \mathrm{GeV} / c$;

- $R_{\mathrm{L}} \geq-2.5$, for $p>1 \mathrm{GeV} / c$.

For electrons with momentum below $1 \mathrm{GeV} / c$, only the first cut is applied, since these electrons do not always reach the ECAL. The efficiency of these identification cuts is $98 \%$, and the electron purity in the selected sample is approximately $28 \%$. The selection of conversion pairs which is described below increases the purity.

A pair-finding algorithm is applied to all combinations of the selected $\mathrm{e}^{+} \mathrm{e}^{-}$pairs in order to find photon conversions. This algorithm finds the point on each track where both helices are parallel in the plane perpendicular to the beam axis (the $x y$-plane). The longitudinal distance $\left(d_{z}\right)$ and transverse distance $\left(d_{x y}\right)$ between these two points are computed. If the tracks overlap in the $x y$-plane, $d_{x y}$ is defined to be negative, otherwise it is taken as positive. The conversion point is taken to lie midway between these two points. The invariant mass of the track pair at the conversion point $\left(m_{\mathrm{ee}}\right)$ and the distance of the point to the beam axis $\left(\rho_{\text {conv }}\right)$ are calculated. The pair is classified as a converted photon if it satisfies:

- $\left|d_{x y}\right| \leq 1.5 \mathrm{~cm}$

- $d_{z} \leq 1.5 \mathrm{~cm}$

- $m_{\mathrm{ee}} \leq 30 \mathrm{MeV} / c^{2}$

- $4 \mathrm{~cm} \leq \rho_{\text {conv }} \leq 100 \mathrm{~cm}$

A total sample of 661226 conversions is obtained with these cuts in the selected hadronic events. According to the Monte Carlo simulation, the purity of this sample is $92 \%$. The background is dominated by $\mathrm{e}^{+} \mathrm{e}^{-}$pairs which do not originate from the same conversion. The energy resolution for converted photons is $\sigma_{E} / E \approx 0.015$, approximately independent of energy.

The measured $\pi^{0}$ production rate depends on the efficiency for photon reconstruction, and for conversions this is essentially proportional to the amount of material between the beam line and the TPC. According to Monte Carlo studies the probability to reconstruct a photon conversion is approximately independent of the radius. The distribution of the conversion radius $\rho_{\text {conv }}$ is shown in Fig. 1. One sees differences between data and Monte Carlo simulation, which points to imperfections of the detector modelling.

In order to accurately determine the amount of material between the beam line and TPC, the photon production rate has been measured both from conversions in this region as well as 
from conversions in the TPC gas. The density of the gas and the conversion probability for photons are well known. A comparison of these photon rates yields a correction factor for the amount of the material between the beam line and the TPC.

Photon conversions in the TPC gas $\left(40 \mathrm{~cm} \leq \rho_{\text {conv }} \leq 100 \mathrm{~cm}\right)$ are selected with the same cuts as described above, resulting in a purity of $87.4 \%$. To enhance the fraction of genuine converted photons in the sample, the photon is required to originate from a $\pi^{0}$ decay by demanding that there also be a high-energy calorimetric photon with an energy greater than $3 \mathrm{GeV}$, and that the invariant mass of this photon and the converted photon be between $100 \mathrm{MeV} / \mathrm{c}^{2}$ and $170 \mathrm{MeV} / c^{2}$. In this way the purity of the selected conversions in the TPC gas is improved to $93.8 \%$.

By comparing the photon rates estimated from the inner and TPC-gas regions, it was found that the integrated amount of material was $(0.7 \pm 1.3$ (stat) \pm 1.4 (sys) $) \%$ too high in the Monte Carlo detector simulation. That is, the Monte Carlo description is in fact consistent with the measurement within the estimated error. The small discrepancy is nevertheless taken into account as a correction factor $C_{\gamma}=1.007 \pm 0.013$ (stat) \pm 0.014 (sys) for the determination of the photon efficiency.

The systematic error arises from uncertainties on the material density in the TPC-gas region $(1.0 \%)$, from the track selection $(0.8 \%)$, from the pair selection $(0.5 \%)$ and from the invariant mass cut $(0.3 \%)$. Adding the statistical error $(1.3 \%)$ and systematic errors in quadrature gives a total error of $1.9 \%$.

\subsection{Calorimetric photons}

Calorimetric photons are reconstructed from neutral clusters in the ECAL. The algorithm employed for photon identification in the ALEPH electromagnetic calorimeter is described in detail elsewhere [12] and a brief summary is given here. A cluster is accepted as a photon candidate if its energy-weighted barycentre is at least $2 \mathrm{~cm}$ away from the closest charged track extrapolation. The photon energy is estimated from the energy collected in the four central towers of a cluster, correcting to the full energy from the parameterization of the shower shape for a single photon in the calorimeter. By using this technique, hadronic background and clustering effects are reduced at the cost of a degraded energy resolution of $\sigma_{E} / E \approx 0.25 / \sqrt{E / \mathrm{GeV}}$.

Photons are required to be well contained in the barrel $(|\cos \theta| \leq 0.72)$ or in the endcap $(0.81 \leq|\cos \theta| \leq 0.95)$ regions of the electromagnetic calorimeter and are required to have an energy of at least $600 \mathrm{MeV}$. Below this energy, the photon detection efficiency is poorly simulated. The simulation improves with increasing photon energy, but it is nevertheless difficult to determine precisely the efficiency even at the highest energies. This is primarily because of overlap between the photon and charged particles, e.g., from the conversion of the partner photon from the $\pi^{0}$. For this reason a correction is applied to the photon efficiency, based on the $\pi^{0}$ rate determined from two conversions (cf. Section 5.2).

\section{$5 \quad$ Measurement of the $\pi^{0}$ rate}

The number of neutral pions in a given momentum bin is determined from the invariant mass distribution of photon pairs. The different possibilities for combining calorimetric and converted photons give three different methods of the $\pi^{0}$ reconstruction. To extract the number of $\pi^{0}$ mesons, the invariant mass distributions for the data and for Monte Carlo are parameterized in terms of a Gaussian function for the signal and a background function $\left(f_{\mathrm{bg}}\right)$, which is fitted between $35 \mathrm{MeV} / c^{2}$ and $400 \mathrm{MeV} / c^{2}$. 
The $\pi^{0}$ cross section was calculated using

$$
\frac{1}{\sigma_{\text {had }}} \frac{\mathrm{d} \sigma}{\mathrm{d} x_{p}}=\frac{1}{N_{\text {had }}} \frac{N_{\pi^{0}}^{\text {rec }}(\text { data })}{\Delta x_{p}} \frac{N_{\pi^{0}}^{\text {gen }}(\mathrm{MC})}{N_{\pi^{0}}^{\text {rec }}(\mathrm{MC})} C_{\text {conv }} C_{\text {calor }},
$$

where $N_{\text {had }}$ is the number of hadronic events, $N_{\pi^{0}}^{\text {rec }}\left(N_{\pi^{0}}^{\text {gen }}\right)$ is the number of reconstructed (generated) $\pi^{0}$ mesons in a bin of width $\Delta x_{p}$, with $x_{p}=p / p_{\text {beam }}$ representing the fraction of the beam momentum carried by the $\pi^{0}$. $C_{\text {conv }}$ is a correction factor for the amount of material in the detector and is given by $C_{\text {conv }}=\left(C_{\gamma}\right)^{n}$, where $n=1(2)$ for $\pi^{0}$ mesons reconstructed from one (two) conversions, and the factor $C_{\gamma}=1.007 \pm 0.019$ as described in Section 4.1. $C_{\text {calor }}$ is a correction function for the calorimeter's photon detection efficiency, and depends on the momentum of the $\pi^{0}$ (cf. Section 5.2).

\section{$5.1 \quad \pi^{0}$ reconstructed from two converted photons}

When the neutral pions are reconstructed from two converted photons, the $\gamma \gamma$ invariant mass is computed in four intervals of $x_{p}$ only, because of the low statistics. The Monte Carlo based efficiencies for both photons are corrected as described in Section 4.1. A three-parameter function is used to describe the background distribution:

$$
f_{\mathrm{bg}}(m)=b_{3} m^{b_{1}} \exp \left(-b_{2} m\right)
$$

The momentum resolution for charged tracks leads to a good mass resolution for the $\pi^{0}$ signal. An example of an invariant mass spectrum is shown in Fig. 2. The measured $x_{p}$ distribution for $\pi^{0}$ mesons is given in Table 1.

The invariant mass spectrum of two converted photons (Fig. 2) has a tail at low masses, which is not well described by the Gaussian curve used to fit the signal. This is understood as the effect of bremsstrahlung from a conversion electron. Although the shapes of the lowmass tails differ somewhat between data and Monte Carlo, the total excess with respect to the Gaussian curve is similar (Fig. 2b). Thus the fitted number of neutral pions is approximately corrected by determining the number of reconstructed Monte Carlo $\pi^{0}$ mesons $\left(N_{\pi^{0}}^{\mathrm{rec}}(\mathrm{MC})\right.$ in equation (1)) by also fitting with a Gaussian function.

A summary of the various contributions to the systematic error is given in Table 2. The following possible sources of systematic uncertainty were investigated:

- The systematic error in the amount of material in the detector is estimated to be $1.9 \%$ (cf. Section 4.1).

- The systematic error in the selection efficiency for conversion pairs was investigated by selecting $\pi^{0}$ mesons via one conversion and one calorimetric photon (cf. Section 5.2). The conversion-pair selection was varied by successively removing each of the individual track selection cuts completely, and in addition by varying each of the pair selection cuts in a reasonable range. The systematic error is estimated from the change in the observed $\pi^{0}$ rate. For the $\pi^{0}$ measurement based on two converted photons, this component of the error is taken twice, conservatively assuming full correlation.

- Errors due to possible deviations of the signal shape from the assumed form are investigated by determining the number of reconstructed $\pi^{0}$ mesons in both data and Monte Carlo directly from the number of $\gamma \gamma$ pairs in the signal range, $100 \mathrm{MeV} / c^{2}$ to $170 \mathrm{MeV} / c^{2}$, and subtracting the fitted background. The relative change in the $\pi^{0}$ rate is small and is taken as the systematic error. 
- Because of the small number of $\pi^{0}$ mesons that can be reconstructed as two conversion pairs, it is necessary to have large momentum bins. The efficiency to find a conversion varies significantly as a function of momentum within a bin, particularly at low momenta. The average efficiency over the bin is estimated by assuming the $\pi^{0}$ momentum spectrum from the Monte Carlo, which leads to a systematic uncertainty from the model dependence. This is estimated by taking the difference between JETSET 7.4 and HeRwig 5.8. This error is only significant in the lowest momentum bin. For the differential cross section obtained using one conversion and one calorimetric photon (Section 5.2), the momentum dependence is obtained directly from the data, so that this uncertainty does not enter into the final result.

\section{$5.2 \quad \pi^{0}$ reconstructed from one converted and one calorimetric photon}

The higher statistics of $\pi^{0}$ mesons reconstructed via one converted and one calorimetric photon allow the measurement of the $\pi^{0}$ spectrum in smaller momentum bins. For $\pi^{0}$ mesons reconstructed via one converted photon and one calorimetric photon or via two calorimetric photons a four-parameter function is used to describe the background:

$$
f_{\mathrm{bg}}(m)=b_{4} m^{b_{1}} \exp \left(-b_{2} m+b_{3} m^{2}\right) .
$$

A typical invariant mass distribution is shown in Fig. 3.

The reconstruction efficiency for calorimetric photons is difficult to estimate with high precision using the Monte Carlo; this is particularly true at low energies. A correction for the efficiency was therefore determined in the following way. The $\pi^{0}$ spectrum was measured in the same four momentum bins as were used in the measurement with two converted photons. Differences between the two measurements are always smaller than $5 \%$, as can be seen from the ratio shown in Fig. 4. As one approaches the extreme case $x_{p}=1$, the $\pi^{0}$ cannot be accompanied by any other high energy particle, which by overlapping with the shower of the photon in the calorimeter would be the main cause of uncertainty in the efficiency. Thus at $x_{p}=1$ it is assumed that the efficiency is correctly simulated by the Monte Carlo. A straight line connecting these points (and the value 1 at $x_{p}=1$ ) is used as an estimate of the influence from imperfections in the detector simulation as a function of the $\pi^{0}$ momentum. This function is applied as a correction to the measurement of the $\pi^{0}$ spectrum via one converted and one calorimetric photon in finer bins $\left(C_{\text {calor }}\right.$ in equation (1)). The error in $C_{\text {calor }}$ is estimated by connecting the error bars of adjacent points in Fig. 4 and the value 1 at $x_{p}=1$. The small variation of the efficiency within the finer bins is taken from the Monte Carlo.

The total number of $\pi^{0}$ mesons is fixed mainly by the measurement via two conversions, while the shape of the spectrum is measured via one conversion and one calorimetric photon. The results are given in Table 3 and are shown in Fig. 5.

The systematic error arises mainly from the measurement via two converted photons because this is used to correct the estimation of the efficiency:

- The systematic error of this correction is explained in Section 5.1; only the first four sources in Table 2 contribute to the systematic error here because for the measurement via one converted photon and one calorimetric photon the error related to the variation of the efficiency within the bins is negligible due to the smaller bin size. The statistical error of this correction corresponds to the number of $\pi^{0}$ mesons reconstructed via two conversions.

- The systematic uncertainty on the efficiency of the electromagnetic calorimeter for photon reconstruction in hadronic events is estimated by the difference to the $\pi^{0}$ analysis with two converted photons. 
The errors are given in Table 4 for the same bins as in Section 5.1.

In the $x_{p}$ range from 0.025 to 1 , one obtains

$$
4.80 \pm 0.07 \text { (stat) } \pm 0.31(\text { sys })
$$

$\pi^{0}$ mesons per hadronic $\mathrm{Z}$ decay. The $\pi^{0}$ spectrum can be extrapolated into the inaccessible momentum range using the shape predicted by the JETSET 7.4 model to calculate the total number of $\pi^{0}$ mesons per $\mathrm{Z}$ decay. This was done by multiplying the measured multiplicity in $0.025<x_{p}<1$ with the ratio of multiplicities $N_{\pi^{0}}\left(0<x_{p}<1\right) / N_{\pi^{0}}\left(0.025<x_{p}<1\right)$ as predicted by the JETSET 7.4 model. The result is $9.63 \pm 0.13$ (stat) \pm 0.62 (sys) \pm 0.12 (extr) $\pi^{0}$ mesons per hadronic $\mathrm{Z}$ decay for the full momentum range. The third error accounts for the systematic uncertainties due to the extrapolation procedure. It has been estimated by determining the extrapolation correction using the HERWIG 5.8 model, and also by varying the JETSET parameters in a reasonable range.

\subsection{1 $\pi^{0}$ production as a function of $p_{\mathrm{T}}^{\text {out }}$ and $p_{\mathrm{T}}^{\text {in }}$}

Neutral pions reconstructed via one converted photon and one calorimetric photon are also used to measure the differential $\pi^{0}$ cross sections as a function of $p_{\mathrm{T}}^{\text {out }}$, the momentum component of the $\pi^{0}$ meson perpendicular to the event plane defined by the sphericity tensor, and $p_{\mathrm{T}}^{\text {in }}$, the momentum component transverse to the sphericity axis and projected onto the event plane. The event plane is determined using both charged and neutral particles. The efficiency corrections determined in Section 5.2 as a function of the momentum are used, and the systematic uncertainty is estimated in the same way. The results for neutral pions and comparisons to the corresponding distributions for charged particles are given in Table 5 and shown in Fig. 6 and Fig. 7.

\section{$5.3 \quad \pi^{0}$ reconstructed from two calorimetric photons}

To provide a cross check on the results presented in Section 5.2, $\pi^{0}$ mesons are reconstructed from two calorimetric photons. Compared to the results obtained with converted photons, the $\gamma \gamma$ invariant mass distributions from two calorimetric photons show several differences. At low $\pi^{0}$ momentum, the energy resolution of electromagnetic showers is rather poor and the efficiency is not as well known as for converted photons. Fluctuations in high energy showers lead to fake photons, mainly at low momentum, which increase the background in the $\pi^{0}$ mass distribution. For high momenta, the photons of the $\pi^{0}$ are often merged, and the signal becomes difficult to extract. Therefore the range in $x_{p}$ of this measurement has been restricted to $0.035<x_{p}<0.6$.

In order to determine corrections to the reconstruction efficiency of calorimetric photons as obtained from the Monte Carlo simulation the following method is used: The number of $\pi^{0}$ mesons is measured using one converted photon and one calorimetric photon (cf. Section 5.2) as a function of the calorimetric photon energy. Differences between data and Monte Carlo simulation could be due to imperfections in the modelling of the efficiency to reconstruct a photon in the ECAL, or an incorrectly generated flux of $\pi^{0}$ mesons. Therefore, these numbers are normalized to the $\pi^{0}$ rate obtained with one high energy calorimetric photon $E_{\gamma}>3 \mathrm{GeV}$, where the ECAL reconstruction efficiency is well understood. Assuming the shape of the momentum spectrum for photons originating from $\pi^{0}$ decays from the Monte Carlo simulation, one obtains a correction for the reconstruction efficiency as the normalized $\pi^{0}$ rate between data and Monte Carlo simulation. This correction depends on the energy of the reconstructed photon and is always smaller than $10 \%$.

The systematic errors for the $\pi^{0}$ measurement via two calorimetric photons arise from several sources: 
- The statistical error on the normalization factor arises from the number of $\pi^{0}$ mesons giving a calorimetric photon of energy larger than $3 \mathrm{GeV}(1.5 \%)$.

- The precision of the energy calibration of calorimetric photons $(2.5 \%)$.

- A possible difference in rates, compared to MC events, between high energy calorimetric photons (where the yield is normalized) and low energy calorimetric photons $(3.6 \%)$. All of these errors are added in quadrature for each calorimetric photon and then counted twice (conservatively assuming full correlation) for the pairs.

- Deviations may occur when the shape of the signal does not exactly match the fitted functions $(2.5 \%)$.

Integrated over the full $x_{p}$ range, the number of $\pi^{0}$ mesons per $\mathrm{Z}$ decay measured via two calorimetric photons is found to be $10.31 \pm 0.03$ (stat) \pm 0.99 (sys), which agrees well with the result in Section 5.2.

\section{Discussion of the results}

Figure 5 shows that the inclusive momentum spectrum of $\pi^{0}$ mesons is well predicted by JETSET 7.4 and HERWIG 5.8 in the low-energy region, whereas at high momentum the Monte Carlo predictions are slightly too high. The $x_{p}$ spectrum reported here is in good agreement with the measurement by the DELPHI experiment [15], which for $x_{p}<0.33$ is based on a similar technique. At high $x_{p}$, however, the DELPHI measurement is based on two calorimetric photons, which results in somewhat larger errors than those reported here.

In Fig. 8 the $\pi^{0}$ spectrum is compared to the charged pion spectrum [1]. At low momenta more neutral pions than charged pions are found, which is well described by the Monte Carlo generators, especially by JETSET 7.4. This excess of neutral pions is mainly due to decays of $\eta$ mesons.

For charged particles with $p_{\mathrm{T}}^{\text {out }}>1 \mathrm{GeV}$, ALEPH [2] found that model predictions for the rate are low by up to $30 \%$. The deviations from JETSET 7.4 predictions obtained for neutral pions are qualitatively the same as for charged particles, so that their ratio is well modelled. HERWIG 5.8 shows a very different behavior. The prediction from HERWIG for the charged particles is also too low, but for the neutral pions the model matches the data very well. As a result the neutral-to-charged ratio is significantly too high, as can be seen in Fig. 6 . The $\pi^{0}$ to charged particle ratio decreases to values less than one because the denominator includes not only $\pi^{ \pm}$, but also, e.g., charged kaons and protons, for which the relative fractions increase as a function of momentum.

For the variable $p_{\mathrm{T}}^{\mathrm{in}}$, both the charged particle and neutral pion distributions are well described by JETSET. HERWIG provides a good description of the charged particle $p_{T}^{\text {in }}$ distribution, but is too high for the neutral pions, leading to the high prediction for the ratio shown in Fig. 7. The discrepancies between data and HERwIG predictions for the $p_{\mathrm{T}}^{\text {in }}$ and $p_{\mathrm{T}}^{\text {out }}$ ratios are difficult to understand, since they occur at relatively high values of $p_{\mathrm{T}}$ (1 to $3 \mathrm{GeV}$ ). Here, one would expect effects that distinguish between charged and neutral hadrons to play a negligible role, which is apparently not the case.

\section{Conclusions}

The inclusive $\pi^{0}$ production is measured in the momentum range $0.025<x_{p}=p / p_{\text {beam }}<1$ with $\pi^{0}$ mesons reconstructed from one photon converted into an $\mathrm{e}^{+} \mathrm{e}^{-}$pair and one photon 
detected in the electromagnetic calorimeter. In this $x_{p}$ range, one obtains

$$
4.80 \pm 0.07 \text { (stat) } \pm 0.31 \text { (sys) }
$$

$\pi^{0}$ mesons per hadronic $\mathrm{Z}$ decay. When extrapolated to the full $\pi^{0}$ momentum range with the Jetset 7.4 Monte Carlo, the integrated $\pi^{0}$ rate per $\mathrm{Z}$ decay is found to be $9.63 \pm 0.13$ (stat) \pm 0.62 (sys) \pm 0.12 (extr). This is in agreement with measurements from L3 [6] and DELPHI [15] and with the predictions from Jetset 7.4 and HeRwig 5.8 (cf. Table 6 and Fig. 9). The large data sample and an accurate modelling of the detector response for conversion pairs and calorimetric photons allow the measurement of the $\pi^{0}$ rate to be extended to higher momenta than previously reported.

Deviations from predictions by JETSET 7.4 obtained in the $p_{\mathrm{T}}^{\text {out }}$ spectrum of neutral pions are very similar to those found for charged particles [2]. The HERwIG model, however, provides a good description of the $p_{\mathrm{T}}^{\text {out }}$ distribution for neutral pions, but not for charged particles.

\section{Acknowledgements}

It is a pleasure to thank our colleagues in the accelerator divisions of CERN for the continued good performance of LEP. Thanks are also due to the technical personnel of the collaborating institutions for their support in constructing and maintaining the ALEPH experiment. Those of the collaboration not from member states thank CERN for its hospitality.

\section{References}

[1] D. Buskulic et al. (ALEPH Collab.), Z. Phys. C66 (1995) 355.

[2] D. Buskulic et al. (ALEPH Collab.), Z. Phys. C55 (1992) 209.

[3] P. Abreu et al. (DELPHI Collab.), Nucl. Phys. B444 (1995) 3.

[4] R. Akers et al. (OPAL Collab.), Z. Phys. C63 (1994) 197.

[5] D. Buskulic et al. (ALEPH Collab.), Phys. Lett. B292 (1992) 210;

ALEPH Collab., Contribution to the 27th International Conference on High Energy Physics, Glasgow (July 1994), EPS535.

[6] M. Acciarri et al. (L3 Collab.), Phys. Lett. B328 (1994) 223;

M. Acciarri et al. (L3 Collab.), Jour. of Phys. G (1994) A70.

[7] D. Buskulic et al. (ALEPH Collab.), Z. Phys. C69 (1995) 379.

[8] P. Abreu et al. (DELPHI Collab.), Phys. Lett. B298 (1993) 236;

P. Abreu et al. (DELPHI Collab.), Z. Phys. C65 (1995) 587.

[9] R. Akers et al. (OPAL Collab.), Z. Phys. C68 (1995) 1.

[10] T. Sjöstrand and M. Bengtson, Comp. Phys. Comm. 43 (1987) 367;

T. Sjöstrand, Comp. Phys. Comm. 82 (1994) 74.

[11] G. Marchesini and B.R. Webber, Nucl. Phys. B310 (1988) 461;

G. Marchesini et al., Comp. Phys. Comm. 67 (1992) 465.

[12] D. Decamp et al. (ALEPH Collab.), Nucl. Instr. Meth. A294 (1990) 121;

D. Buskulic et al. (ALEPH Collab.), Nucl. Instr. Meth. A360 (1995) 481. 
[13] D. Decamp et al. (ALEPH Collab.), Z. Phys. C53 (1992) 1.

[14] J.E. Campagne and R. Zitoun, Z. Phys. C43 (1989) 469.

[15] W. Adam et. al (DELPHI Collab.), CERN-PPE/95-144, to be published in Z. Phys. C.

[16] Y.I. Azimov, Yu.L. Dokshitzer, V.A. Khoze and S.I. Troyan, Z. Phys. C27 (1985) 65;

Y.I. Azimov, Yu.L. Dokshitzer, V.A. Khoze and S.I. Troyan, Z. Phys. C31 (1986) 213.

[17] D. Buskulic et al. (ALEPH Collab.), Phys. C69 (1995) 393. 
Table 1: Measured multiplicities and differential cross sections for $\pi^{0}$ production via two conversions. The $\left\langle x_{p}\right\rangle$ values are obtained from Monte Carlo.

\begin{tabular}{|l|c|c|c|}
\hline$x_{p}$ range & $\left\langle x_{p}\right\rangle$ & $\frac{\text { Multiplicity } \pi^{0}}{\mathrm{Z} \text { event }} \pm \Delta_{\text {stat }} \pm \Delta_{\text {sys }}$ & $\frac{1}{\sigma_{\text {had }}} \frac{\mathrm{d} \sigma}{\mathrm{d} x_{p}} \pm \Delta_{\text {stat }} \pm \Delta_{\text {sys }}$ \\
\hline \hline $0.025-0.085$ & 0.061 & $3.288 \pm 0.170 \pm 0.191$ & $54.80 \pm 2.84 \pm 3.18$ \\
$0.085-0.140$ & 0.110 & $0.774 \pm 0.041 \pm 0.036$ & $14.06 \pm 0.75 \pm 0.66$ \\
$0.140-0.200$ & 0.167 & $0.360 \pm 0.026 \pm 0.019$ & $6.00 \pm 0.43 \pm 0.31$ \\
$0.200-1.000$ & 0.301 & $0.336 \pm 0.022 \pm 0.020$ & $0.420 \pm 0.027 \pm 0.025$ \\
\hline
\end{tabular}

Table 2: Systematic errors for $\pi^{0}$ mesons reconstructed via two converted photons. All values are expressed in percent.

\begin{tabular}{|l|c||c|c|c|c|}
\hline Source of error & \multicolumn{5}{|c|}{$x_{p}$ interval } \\
& $0.025-1$. & $0.025-0.085$ & $0.085-0.140$ & $0.14-0.20$ & $0.20-1$. \\
\hline \hline Material density & 3.8 & 3.8 & 3.8 & 3.8 & 3.8 \\
Track selection & 2.9 & 3.5 & 2.6 & 2.7 & 4.0 \\
Pair selection & 1.6 & 2.1 & 0.7 & 1.9 & 1.1 \\
Shape of the $\pi^{0}$ signal & 0.2 & 0.0 & 0.4 & 1.1 & 2.1 \\
$\pi^{0}$ momentum bins & 1.1 & 1.5 & 0.1 & 0.0 & 0.0 \\
\hline \hline Systematic error & & & & & \\
(quadrature total) & 5.2 & 5.8 & 4.7 & 5.2 & 6.0 \\
\hline
\end{tabular}


Table 3: Measured multiplicities and differential cross sections for $\pi^{0}$ production. The $<x_{p}>$ values are obtained from Monte Carlo. The results of summing over the measured $x_{p}$ intervals are also given together with the extrapolation to the full $x_{p}$ range.

\begin{tabular}{|l|c|c|cc|}
\hline$x_{p}$ range & $\left\langle x_{p}\right\rangle$ & $\frac{\text { Multiplicity } \pi^{0}}{\mathrm{Z} \text { event }} \pm \Delta_{\text {stat }} \pm \Delta_{\text {sys }}$ & $\frac{1}{\sigma_{\mathrm{had}}} \frac{\mathrm{d} \sigma}{\mathrm{d} x_{p}} \pm \Delta_{\text {stat }} \pm \Delta_{\text {sys }}$ \\
\hline \hline $0.025-0.035$ & 0.031 & $1.051 \pm 0.059 \pm 0.081$ & $105.1 \pm 5.9 \pm 8.1$ \\
$0.035-0.045$ & 0.040 & $0.701 \pm 0.021 \pm 0.054$ & $70.13 \pm 2.10 \pm 5.40$ \\
$0.045-0.055$ & 0.050 & $0.550 \pm 0.013 \pm 0.042$ & $55.04 \pm 1.33 \pm 4.24$ \\
$0.055-0.070$ & 0.062 & $0.594 \pm 0.011 \pm 0.046$ & $39.63 \pm 0.72 \pm 3.05$ \\
$0.070-0.085$ & 0.077 & $0.400 \pm 0.007 \pm 0.031$ & $26.67 \pm 0.47 \pm 2.05$ \\
$0.085-0.100$ & 0.092 & $0.298 \pm 0.005 \pm 0.022$ & $19.88 \pm 0.36 \pm 1.47$ \\
$0.100-0.120$ & 0.110 & $0.283 \pm 0.004 \pm 0.021$ & $14.15 \pm 0.22 \pm 1.05$ \\
$0.120-0.140$ & 0.130 & $0.210 \pm 0.004 \pm 0.016$ & $10.51 \pm 0.18 \pm 0.78$ \\
$0.140-0.160$ & 0.150 & $0.153 \pm 0.003 \pm 0.014$ & $7.64 \pm 0.14 \pm 0.68$ \\
$0.160-0.180$ & 0.170 & $0.116 \pm 0.002 \pm 0.010$ & $5.79 \pm 0.12 \pm 0.51$ \\
$0.180-0.200$ & 0.190 & $0.0895 \pm 0.0020 \pm 0.0080$ & $4.47 \pm 0.10 \pm 0.40$ \\
$0.200-0.225$ & 0.212 & $0.0860 \pm 0.0019 \pm 0.0090$ & $3.44 \pm 0.08 \pm 0.36$ \\
$0.225-0.250$ & 0.237 & $0.0609 \pm 0.0015 \pm 0.0064$ & $2.44 \pm 0.06 \pm 0.26$ \\
$0.250-0.275$ & 0.262 & $0.0469 \pm 0.0013 \pm 0.0049$ & $1.88 \pm 0.05 \pm 0.20$ \\
$0.275-0.300$ & 0.287 & $0.0342 \pm 0.0011 \pm 0.0036$ & $1.37 \pm 0.04 \pm 0.14$ \\
$0.300-0.350$ & 0.323 & $0.0458 \pm 0.0013 \pm 0.0040$ & $0.917 \pm 0.025 \pm 0.080$ \\
$0.350-0.400$ & 0.373 & $0.0310 \pm 0.0011 \pm 0.0026$ & $0.620 \pm 0.021 \pm 0.052$ \\
$0.400-0.450$ & 0.423 & $0.0173 \pm 0.0008 \pm 0.0014$ & $0.347 \pm 0.016 \pm 0.028$ \\
$0.450-0.500$ & 0.474 & $0.0122 \pm 0.0009 \pm 0.0010$ & $0.245 \pm 0.019 \pm 0.019$ \\
$0.500-0.550$ & 0.523 & $0.0076 \pm 0.0007 \pm 0.0006$ & $0.153 \pm 0.013 \pm 0.011$ \\
$0.550-0.600$ & 0.573 & $0.0051 \pm 0.0004 \pm 0.0004$ & $0.102 \pm 0.009 \pm 0.007$ \\
$0.600-0.700$ & 0.643 & $0.0050 \pm 0.0005 \pm 0.0003$ & $0.050 \pm 0.005 \pm 0.003$ \\
$0.700-1.000$ & 0.770 & $0.0023 \pm 0.0005 \pm 0.0001$ & $0.008 \pm 0.002 \pm 0.001$ \\
\hline \hline $0.025-1.000$ & & $4.802 \pm 0.066 \pm 0.307$ & & \\
\hline \hline all $x_{p}$ & & $9.630 \pm 0.132 \pm 0.616$ & & \\
\hline
\end{tabular}

Table 4: Systematic errors for $\pi^{0}$ mesons reconstructed via one converted photon and one calorimetric photon. All values are expressed in percent.

\begin{tabular}{|l|c||c|c|c|c|}
\hline Source of error & \multicolumn{5}{|c|}{$x_{p}$ interval } \\
& $0.025-1$. & $0.025-0.085$ & $0.085-0.140$ & $0.14-0.20$ & $0.20-1$. \\
\hline \hline Normalization: & & & & & \\
- systematic error & 5.0 & 5.6 & 4.7 & 5.2 & 6.0 \\
- statistical error & 3.8 & 5.2 & 5.3 & 7.2 & 6.6 \\
ECAL efficiency & 0.9 & 0.5 & 2.2 & 0.6 & 0.6 \\
\hline \hline Systematic error & & & & & \\
(quadrature total) & 6.4 & 7.7 & 7.4 & 8.9 & 8.9 \\
\hline
\end{tabular}


Table 5: Measured multiplicities and differential cross sections for $\pi^{0}$ production as a function of $p_{\mathrm{T}}^{\text {out }}$ and $p_{\mathrm{T}}^{\text {in }}$. The $<p_{\mathrm{T}}^{\text {in }}>$ and $<p_{\mathrm{T}}^{\text {out }}>$ values are obtained from Monte Carlo.

\begin{tabular}{|c|c|c|c|cc|}
\hline $\begin{array}{c}p_{\mathrm{T}}^{\text {out }}, p_{\mathrm{T}}^{\text {in }} \text { range } \\
{[\mathrm{GeV} / c]}\end{array}$ & $\left\langle p_{\mathrm{T}}^{\text {out }}\right\rangle$ & $\frac{1}{\sigma_{\text {had }}} \frac{\mathrm{d} \sigma}{\mathrm{d} p_{\mathrm{T}}^{\text {out }}} \pm \Delta_{\text {stat }} \pm \Delta_{\text {sys }}$ & $\begin{array}{c}\left\langle p_{\mathrm{T}}^{\text {in }}\right\rangle \\
{[\mathrm{GeV} / c]}\end{array}$ & $\frac{1}{\sigma_{\text {had }}} \frac{\mathrm{d} \sigma}{\mathrm{d} p_{\mathrm{T}}^{\text {in }}} \pm \Delta_{\text {stat }} \pm \Delta_{\text {sys }}$ \\
\hline $0.00-0.05$ & 0.025 & $36.92 \pm 0.56 \pm 2.36$ & 0.025 & $27.79 \pm 0.52 \pm 1.78$ \\
$0.05-0.10$ & 0.075 & $30.72 \pm 0.48 \pm 1.97$ & 0.075 & $23.75 \pm 0.44 \pm 1.52$ \\
$0.10-0.15$ & 0.125 & $25.99 \pm 0.38 \pm 1.66$ & 0.125 & $21.93 \pm 0.39 \pm 1.40$ \\
$0.15-0.20$ & 0.175 & $21.87 \pm 0.32 \pm 1.40$ & 0.175 & $17.37 \pm 0.32 \pm 1.11$ \\
$0.20-0.25$ & 0.225 & $16.51 \pm 0.24 \pm 1.06$ & 0.225 & $14.42 \pm 0.27 \pm 0.92$ \\
$0.25-0.30$ & 0.275 & $13.24 \pm 0.21 \pm 0.85$ & 0.275 & $11.88 \pm 0.22 \pm 0.76$ \\
$0.30-0.35$ & 0.325 & $10.58 \pm 0.16 \pm 0.68$ & 0.325 & $9.79 \pm 0.19 \pm 0.63$ \\
$0.35-0.40$ & 0.375 & $8.01 \pm 0.12 \pm 0.51$ & 0.375 & $8.75 \pm 0.16 \pm 0.56$ \\
$0.40-0.45$ & 0.425 & $6.36 \pm 0.10 \pm 0.41$ & 0.425 & $6.54 \pm 0.13 \pm 0.42$ \\
$0.45-0.50$ & 0.475 & $5.00 \pm 0.08 \pm 0.32$ & 0.475 & $5.99 \pm 0.12 \pm 0.39$ \\
$0.50-0.60$ & 0.548 & $3.28 \pm 0.05 \pm 0.21$ & 0.549 & $4.67 \pm 0.07 \pm 0.30$ \\
$0.60-0.70$ & 0.648 & $2.14 \pm 0.03 \pm 0.14$ & 0.649 & $3.34 \pm 0.05 \pm 0.21$ \\
$0.70-0.80$ & 0.748 & $1.22 \pm 0.02 \pm 0.08$ & 0.749 & $2.53 \pm 0.04 \pm 0.16$ \\
$0.80-0.90$ & 0.847 & $0.801 \pm 0.015 \pm 0.051$ & 0.849 & $1.94 \pm 0.04 \pm 0.12$ \\
$0.90-1.00$ & 0.948 & $0.519 \pm 0.012 \pm 0.033$ & 0.949 & $1.57 \pm 0.03 \pm 0.10$ \\
$1.00-1.50$ & 1.194 & $0.181 \pm 0.003 \pm 0.012$ & 1.221 & $0.845 \pm 0.009 \pm 0.054$ \\
$1.50-2.00$ & 1.709 & $0.039 \pm 0.001 \pm 0.003$ & 1.727 & $0.352 \pm 0.005 \pm 0.023$ \\
$2.00-2.50$ & 2.216 & $0.012 \pm 0.001 \pm 0.001$ & 2.229 & $0.173 \pm 0.003 \pm 0.011$ \\
$2.50-3.00$ & 2.717 & $0.004 \pm 0.001 \pm 0.001$ & 2.729 & $0.094 \pm 0.002 \pm 0.006$ \\
\hline
\end{tabular}

Table 6: Measured multiplicities for $\pi^{0}$ mesons extrapolated to the full $x_{p}$ range.

\begin{tabular}{|l|l|}
\hline ALEPH & $9.63 \pm 0.13 \pm 0.62 \pm 0.12$ \\
DELPHI & $9.2 \pm 0.2 \pm 1.0$ \\
L3 & $9.18 \pm 0.03 \pm 0.73$ \\
\hline JETSET 7.4 & 9.414 \\
HERWIG 5.8 & 9.615 \\
\hline
\end{tabular}




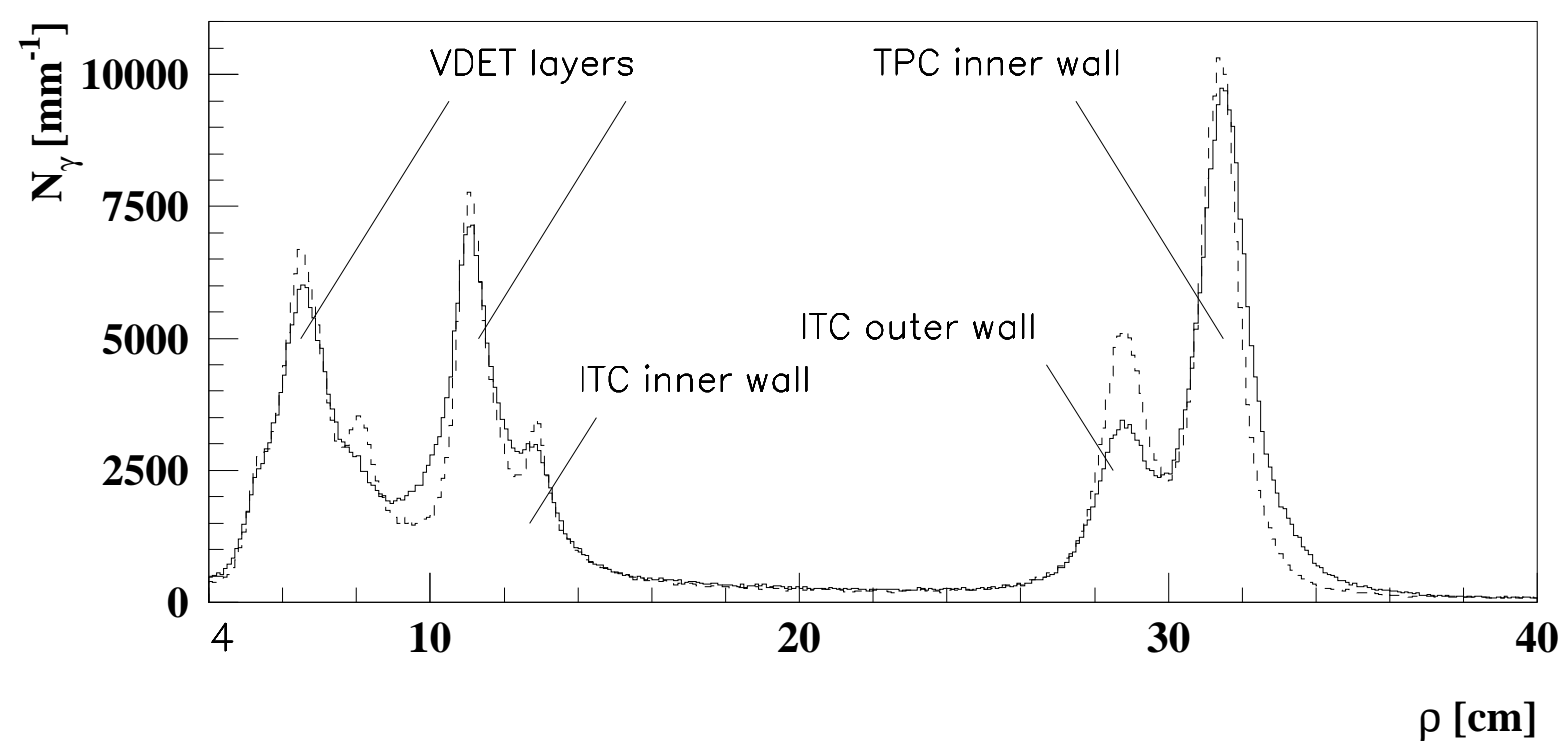

Figure 1: Conversion radius distribution in data (full line) and Monte Carlo simulation (dashed line)
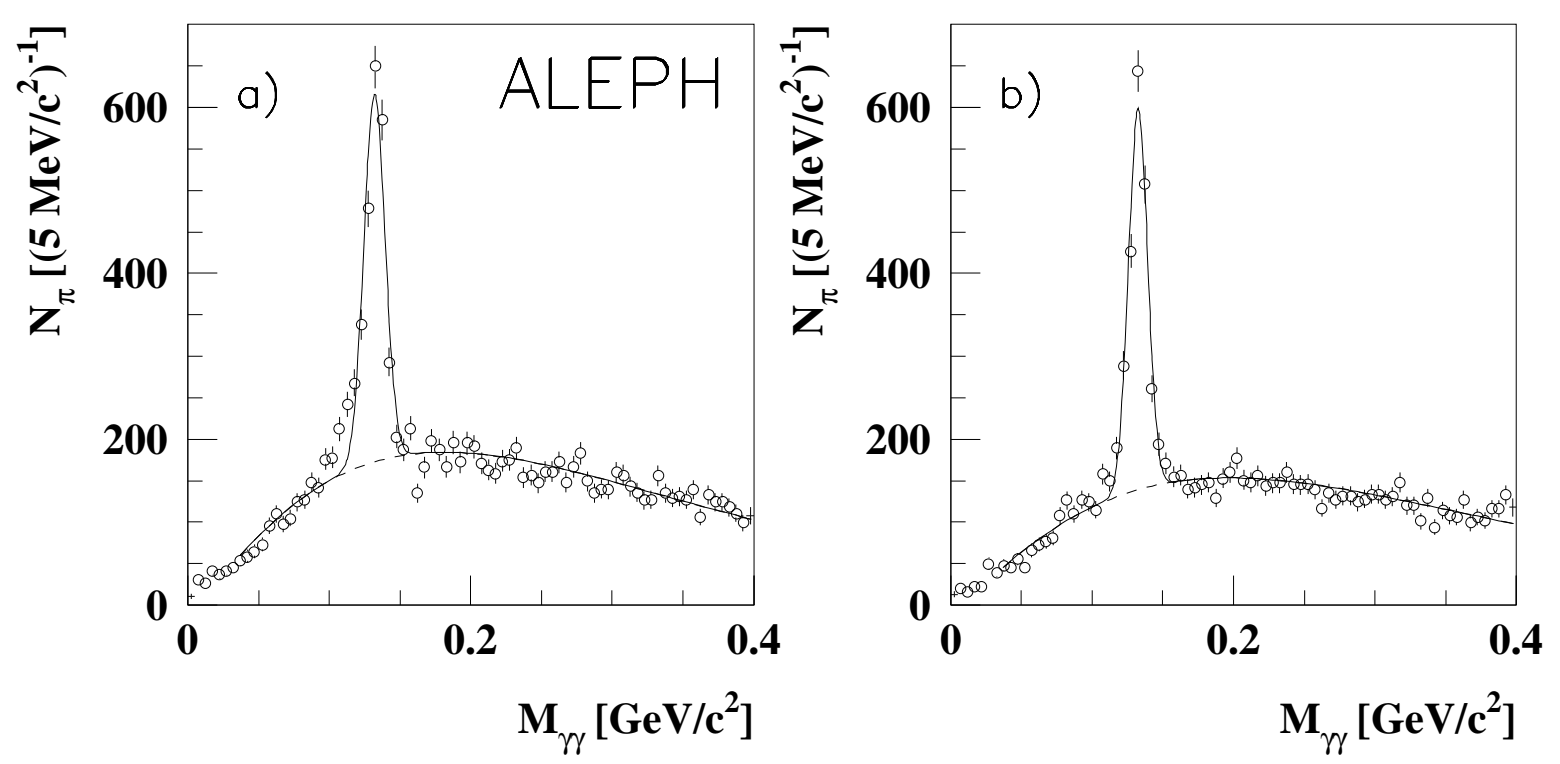

Figure 2: Invariant $\gamma \gamma$ mass distribution for combinations of two conversions in the data (a) and in the Monte Carlo (b) in the $x_{p}$ range $0.025-0.085$. The full lines represent the result of the fits using a sum of a Gaussian function and the parameterization for the background, which is plotted as dashed line. 

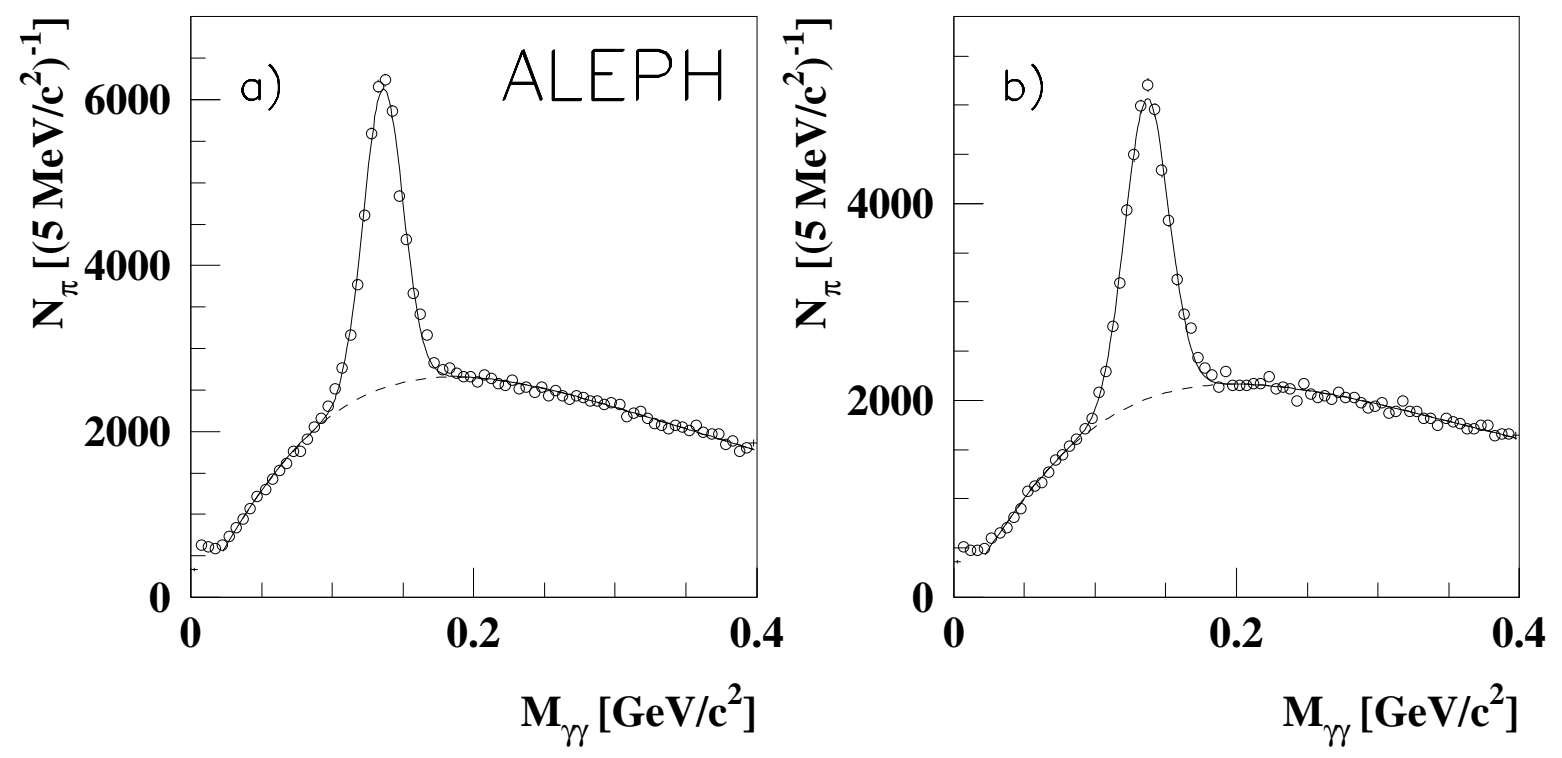

Figure 3: Invariant $\gamma \gamma$ mass distribution for combinations of one conversion and one calorimetric photon in the data (a) and in the Monte Carlo (b) in the $x_{p}$ range $0.085-0.1$. The full lines represent the result of the fits using a sum of a Gaussian function and the parameterization for the background, which is plotted as dashed line.

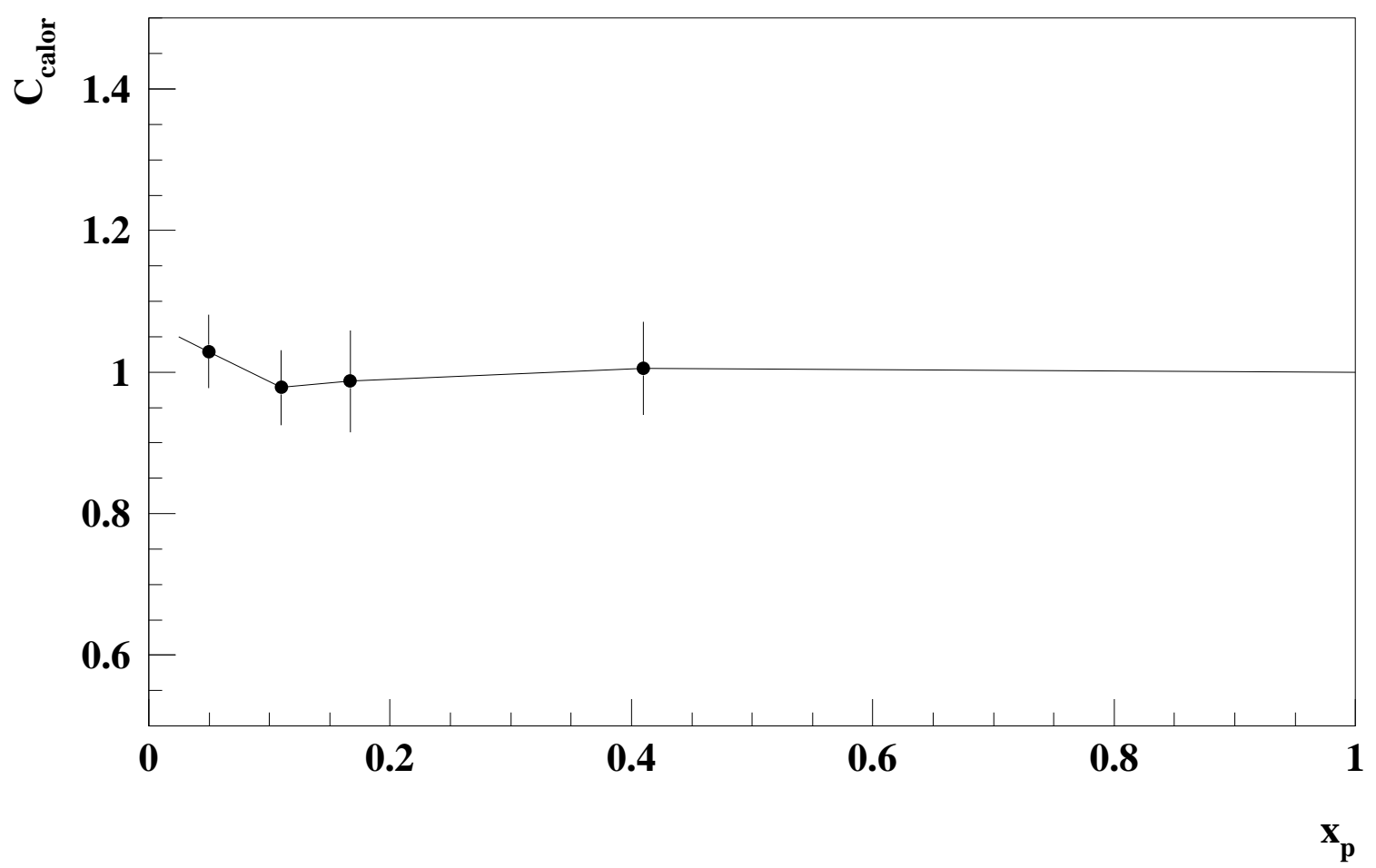

Figure 4: Ratio of $\pi^{0}$ production measured via two conversions over $\pi^{0}$ production measured via one conversion and one calorimetric photon as function of $x_{p}$. 


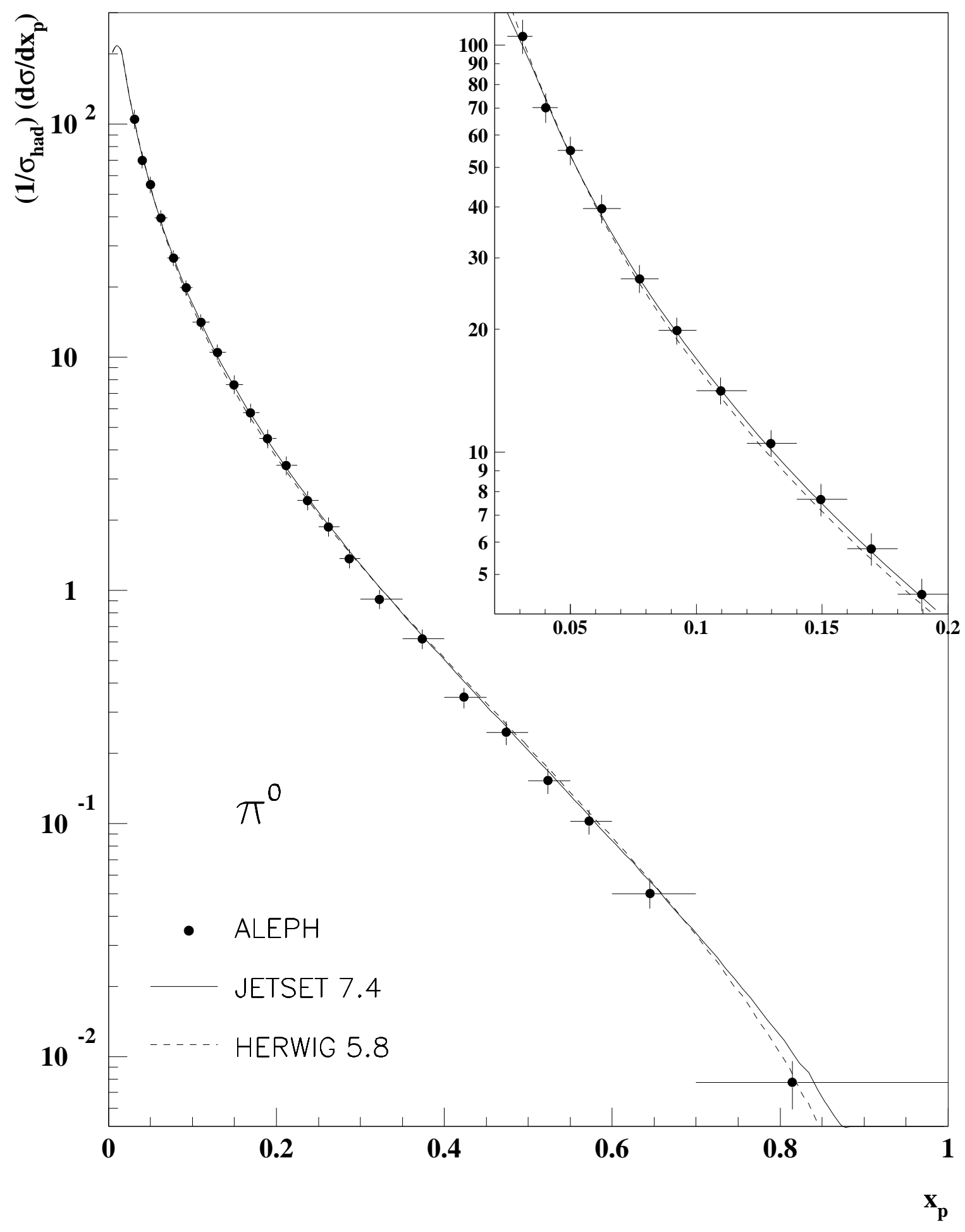

Figure 5: Differential inclusive $\pi^{0}$ cross section as a function of $x_{p}$. The error bars show the total errors (statistical and systematic errors added in quadrature). 

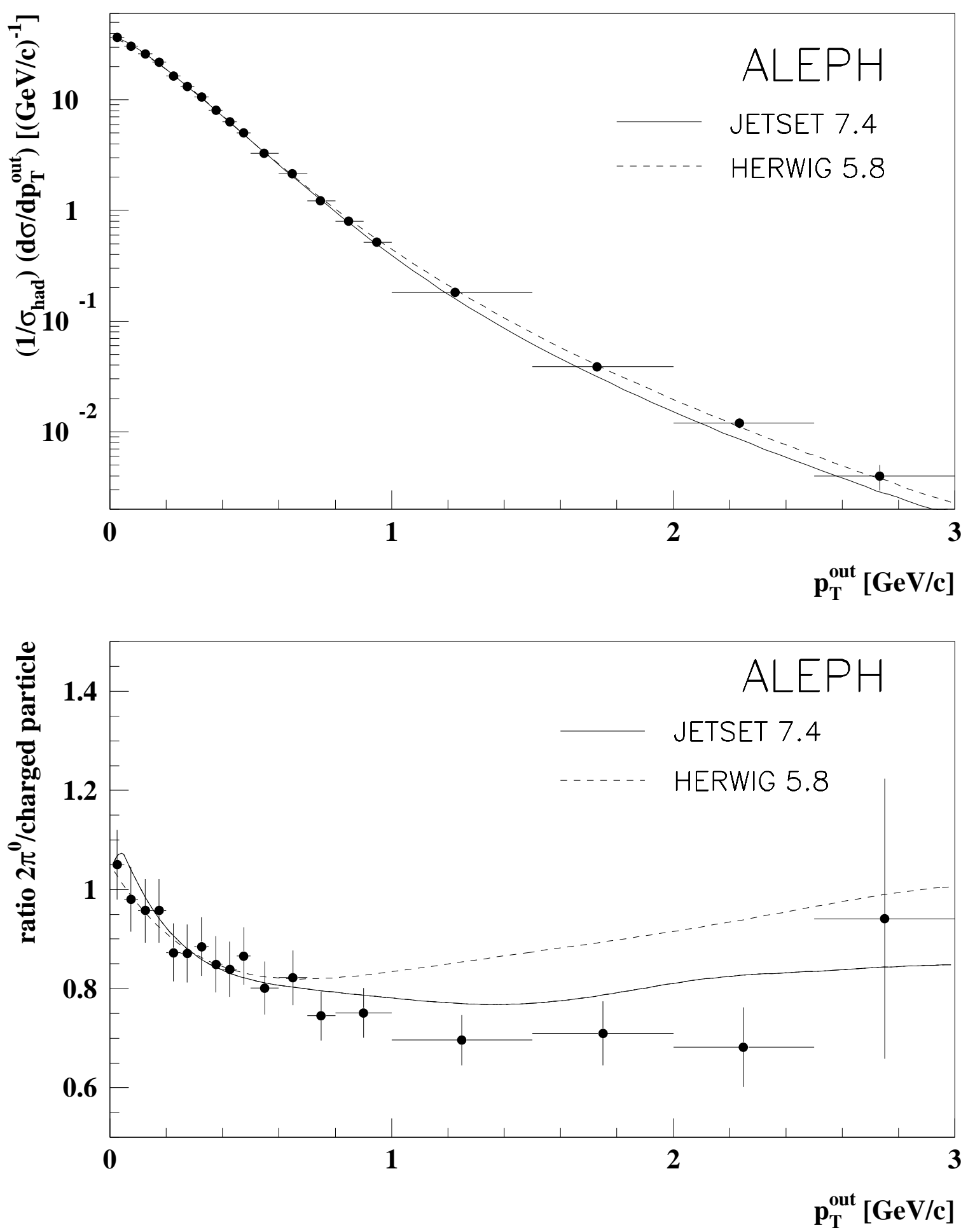

Figure 6: Differential inclusive $\pi^{0}$ cross section and the ratio of neutral pions per charged particle as a function of $p_{\mathrm{T}}^{\text {out }}$. The error bars show the total errors (statistical and systematic errors added in quadrature). 

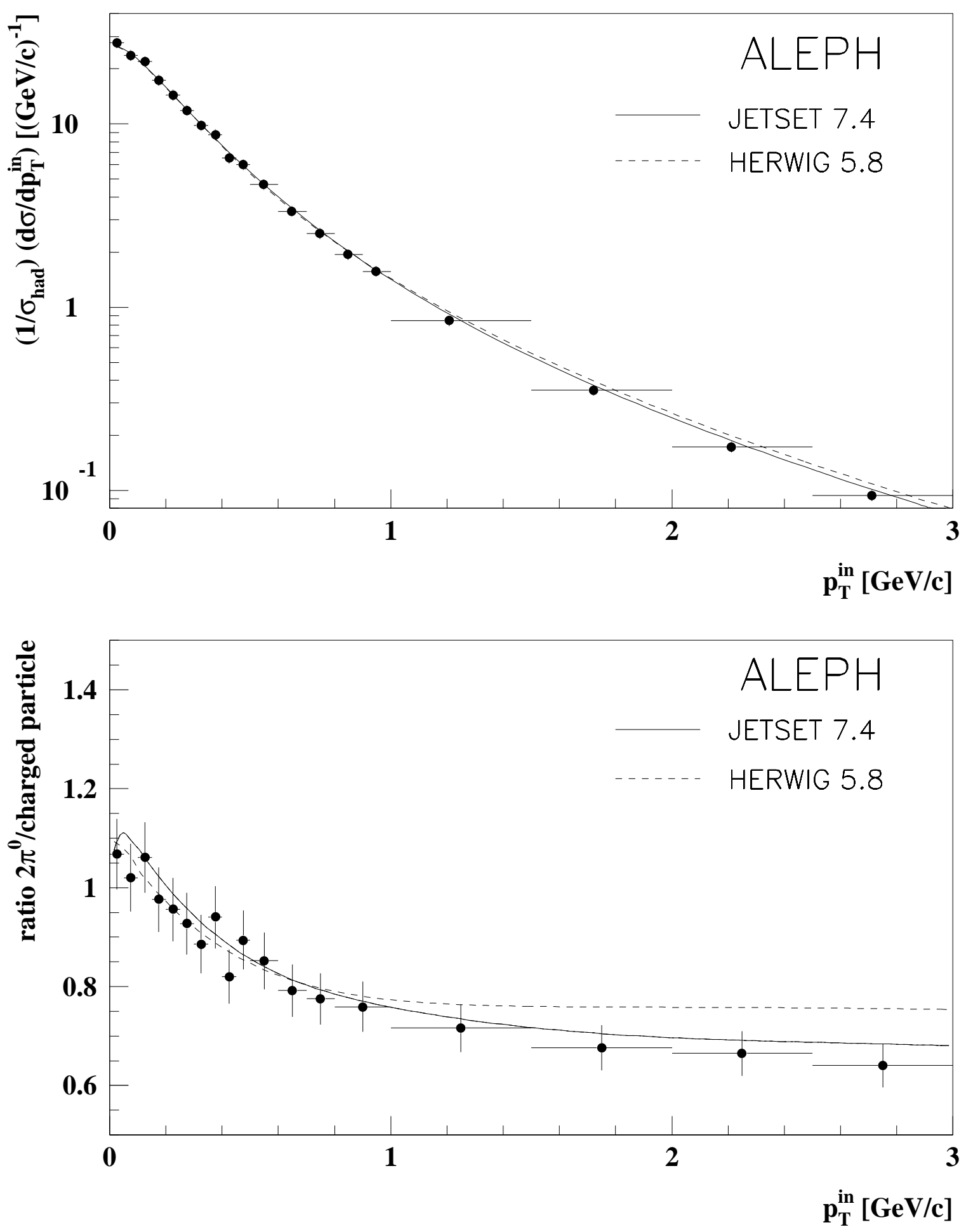

Figure 7: Differential inclusive $\pi^{0}$ cross section and the ratio of neutral pions per charged particle as a function of $p_{\mathrm{T}}^{\mathrm{in}}$. The error bars show the total errors (statistical and systematic errors added in quadrature). 

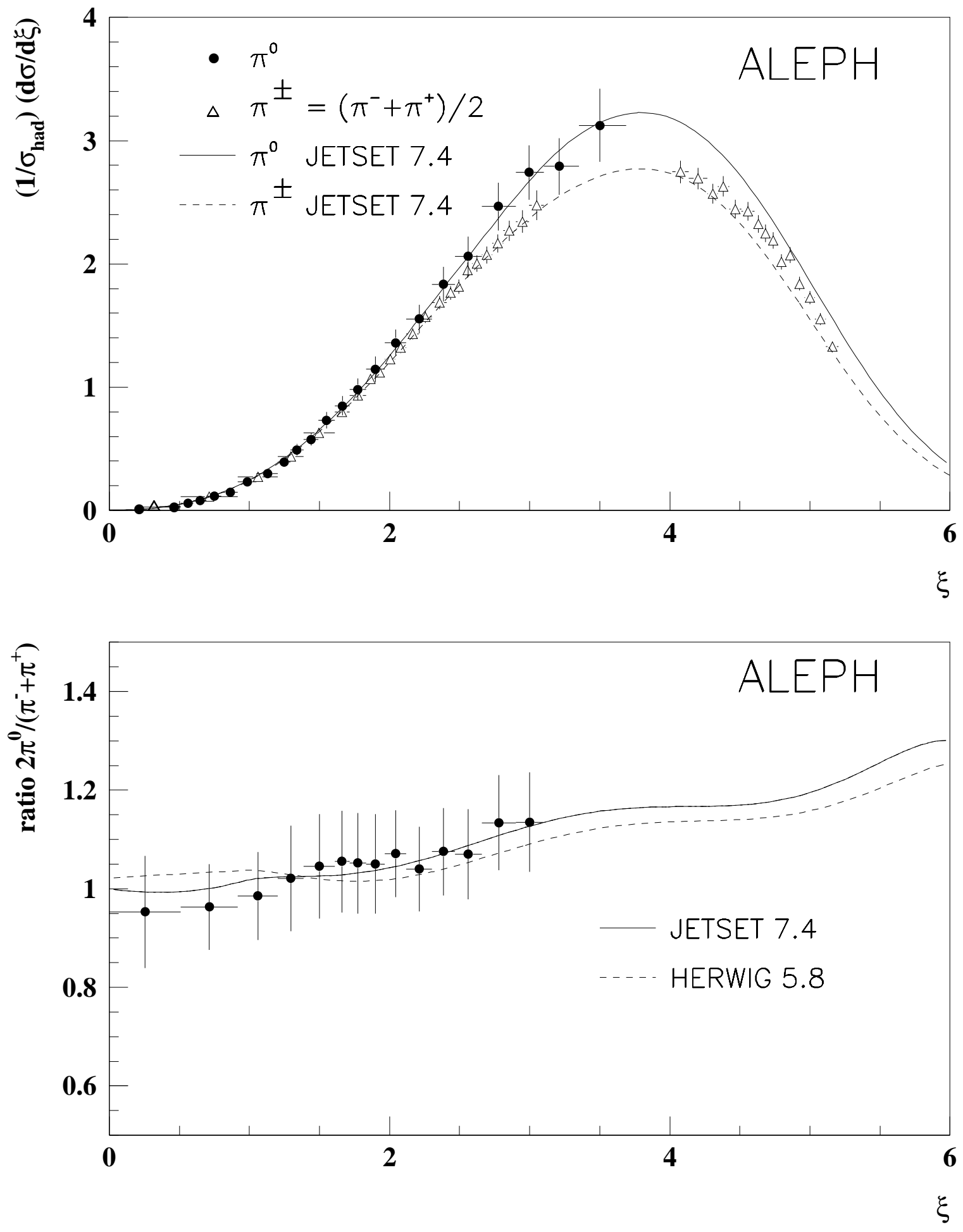

Figure 8: Differential inclusive cross section of neutral and charged pions and their ratio as a function of $\xi=\ln \left(1 / x_{p}\right)$. The error bars show the total errors (statistical and systematic errors added in quadrature). 


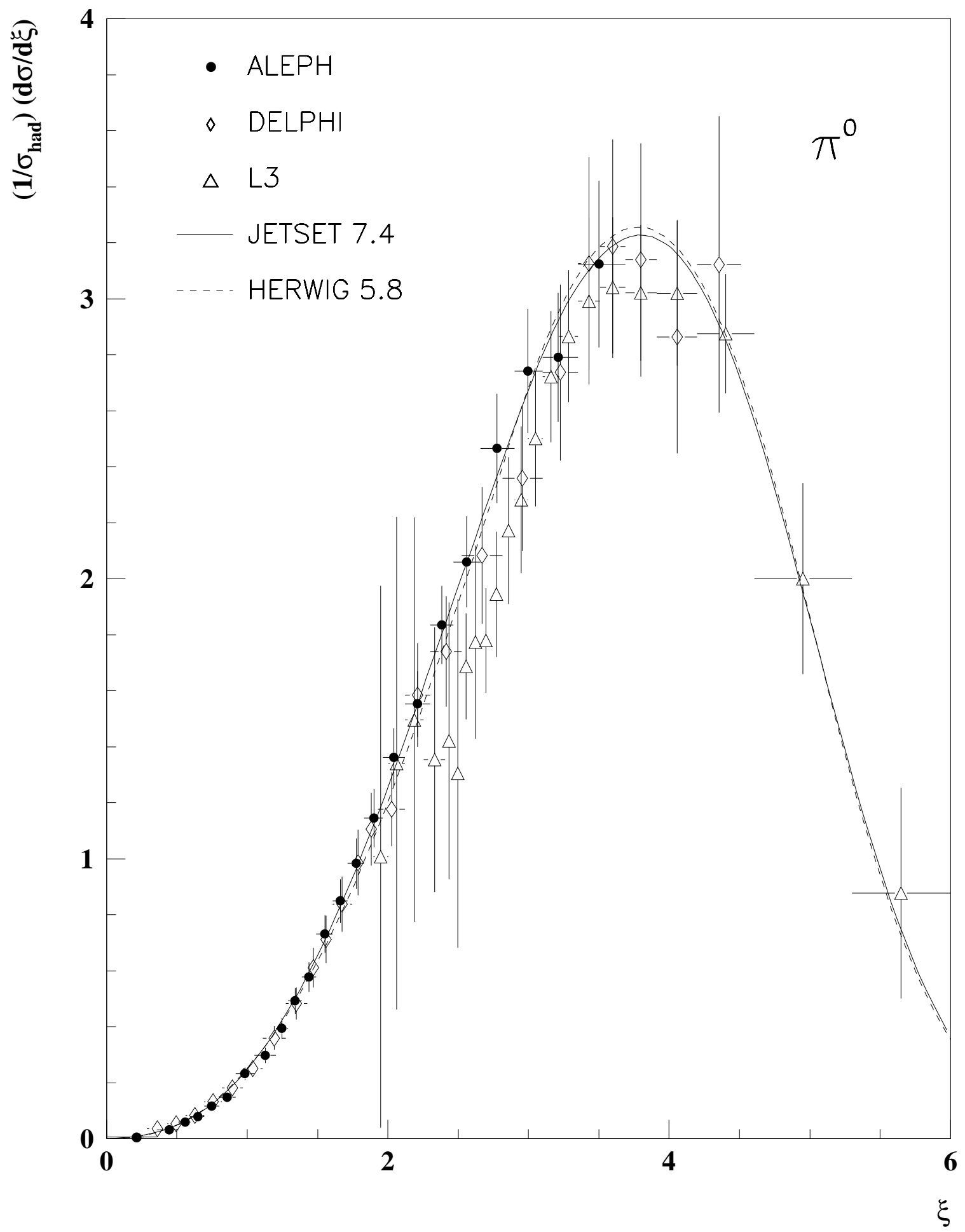

Figure 9: Differential inclusive $\pi^{0}$ cross section as a function of $\xi=\ln \left(1 / x_{p}\right)$ measured by ALEPH, DELPHI and L3. The error bars show the total errors (statistical and systematic errors added in quadrature). 